\title{
Ewing's Sarcoma of Maxilla: A Rare Presentation
}

${ }^{1}$ SM Azeem Mohiyuddin, ${ }^{2}$ Ravindra P Deo, ${ }^{3}$ Divya N Jyothi, ${ }^{4}$ Harendra ML Kumar, ${ }^{5}$ A Sagayaraj

\begin{abstract}
Ewing's sarcoma is a rare malignant small cell tumor occurring in the first and second decades of life and usually involving the long bones of the limb. Literature shows that less than $3 \%$ of the tumor originates in the maxillofacial region of which $1 \%$ occurs in jaw bones with higher predilection for mandible. Mean age of presentation of this tumor in bones of head and neck region is 10.9 years.

Surgery is the first line of treatment and considering the aggressive behavior of the tumor multimodality treatment is preferred to reduce recurrence rate.

Due to small number of cases affecting the jaws, there are no definite protocols in treatment of Ewing's sarcoma of maxilla.

We are presenting a case of Ewing's sarcoma of maxilla in a 60 years old patient, as it is involving a rare site and has occurred in an uncommon age group.
\end{abstract}

Keywords: Ewing's sarcoma, Maxilla, Maxillectomy, Malignancy.

How to cite this article: Mohiyuddin SMA, Deo RP, Jyothi DN, Kumar HML, Sagayaraj A. Ewing's Sarcoma of Maxilla: A Rare Presentation. Int J Head Neck Surg 2015;6(2):96-98.

Source of support: Nil

Conflict of interest: None

\section{INTRODUCTION}

Ewing's sarcoma family of tumor (ESFT) includes Ewing's sarcoma, peripheral primitive neuroectodermal tumor, neuroepithelioma and Askin tumor (tumor of the chest wall). ${ }^{1,2}$ Ewing's sarcoma family of tumor is thought to be derived from cells of the neural crest and are found to contain the same reciprocal translocation between chromosome 11 and 22,t(11;22). ${ }^{1}$

Ewing's sarcoma accounts for approximately $6 \%$ of all the malignant bone tumors. Approximately, $4 \%$ of Ewing's sarcoma cases have arisen in bones of head and neck, with $1 \%$ occurring in jaws. When jaw is involved,

\footnotetext{
${ }^{1,4}$ Professor and Head, ${ }^{2}$ Visiting Professor

${ }^{3}$ Senior Resident, ${ }^{5}$ Associate Professor

1-3,5 Department of Otorhinolaryngology and Head and Neck Surgery Sri Devaraj Urs Medical College, Kolar, Karnataka, India

${ }^{4}$ Department of Pathology, Sri Devaraj Urs Medical College, Kolar Karnataka, India
}

Corresponding Author: SM Azeem Mohiyuddin, Professor and Head, Department of Otorhinolaryngology and Head and Neck Surgery, Sri Devaraj Urs Medical College, Kolar, Karnataka India, Phone: 09845373279, e-mail: azeem_hn@yahoo.co.in the predilection is for the ramus of mandible, with only a few cases reported in maxilla. ${ }^{3}$ Primary maxilla involvement is rare, and only 30 cases have been reported so far. ${ }^{4}$ Ewing's sarcoma commonly presents in children; incidence after the 3 rd decade accounts for only $10 \%$ of cases and is limited to only a few case reports in patients older than 60 years. A male preponderance is seen. ${ }^{5}$

Here, we are reporting a case of Ewing's sarcoma primarily affecting the maxilla in a 60-year-old man.

\section{CASE REPORT}

A 60-year-old man reported to our department with complaints of right-sided facial pain and swelling of 1 month duration. He had right-sided nasal obstruction since 2 weeks and bleeding from right nasal cavity since 8 days. Clinical examination showed an irregular fleshy mass in the right nasal cavity at the level of middle meatus, which bled on touch and could not be probed laterally. There was a solitary, spherical, smooth, tender, firm, immobile swelling approximately $2 \times 3 \mathrm{~cm}$, in the region of canine fossa (Fig. 1). Medial half of the infraorbital rim was not palpable and there was fullness in the right medial canthal region.

Intraoral examination showed a smooth soft swelling in the upper gingivobuccal sulcus at the level of canine tooth extending up to the 1st molar. Mucosa over the swelling was normal.

There was mild proptosis of the right eye with epiphora; eye movements were normal in all directions. He had paresthesia over right infraorbital region.

A computed tomography (CT) scan showed lobular soft tissue density lesion in right maxillary sinus causing bony erosion of all the walls of the sinus with extension of the lesion into the right orbit, nasal cavity, premaxillary region and infratemporal fossa (Fig. 1).

Patient underwent diagnostic nasal endoscopy and biopsy, which was reported as neuroectodermal tumor. Preoperative work up was done and patient underwent right total maxillectomy and the entire tumor was removed en bloc maintaining adequate margins (Fig. 2). Patient made uneventful recovery postoperatively.

Histopathological report showed cells arranged in nests, alveolar pattern and discohesive. These cells were small uniform round cells with high nucleocytoplasmic ratio, vesicular nucleus with fine chromatin, with 


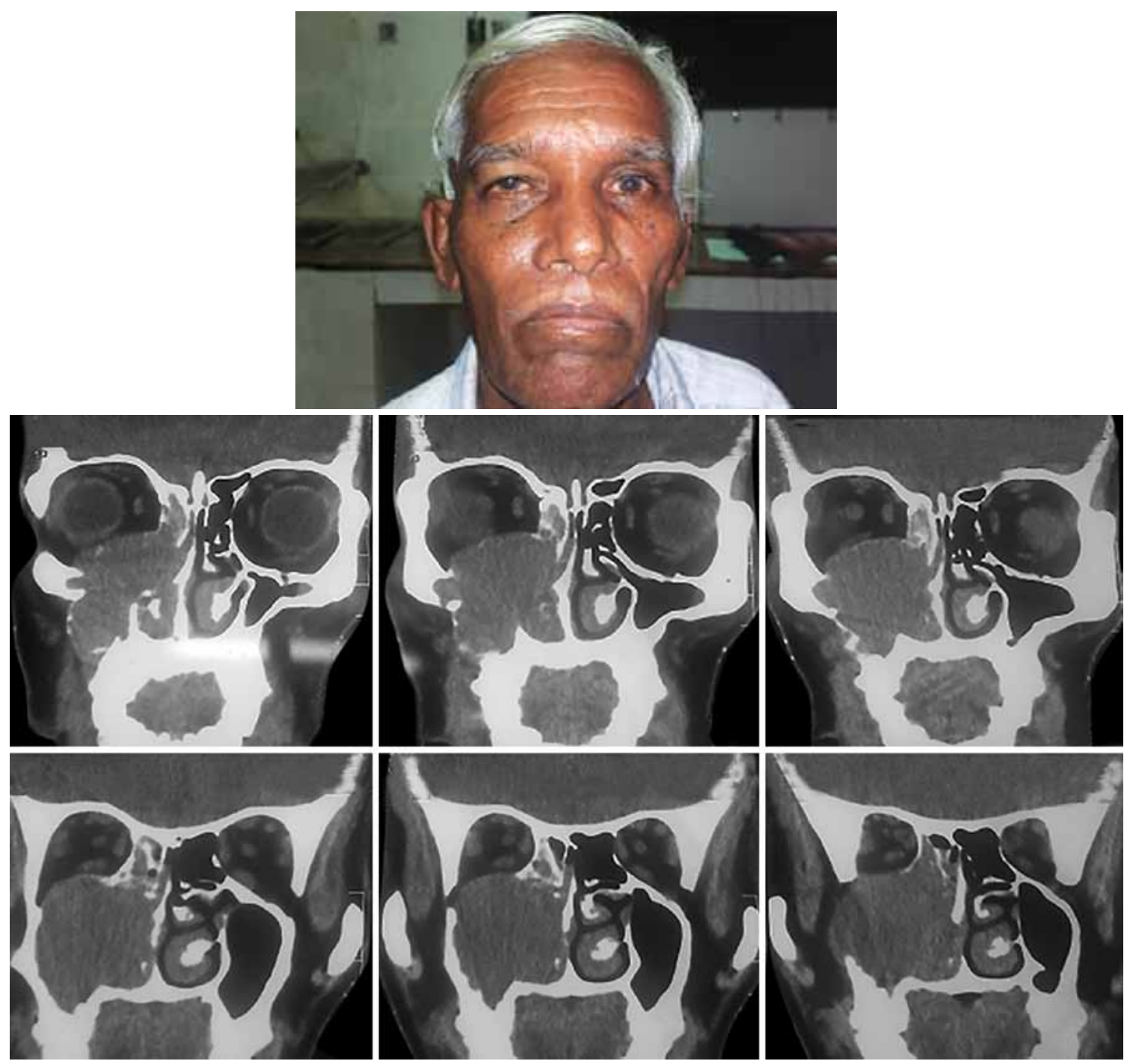

Fig. 1: Patient showing fullness over right maxillary region and asymmetry of the eyes with the CT scan of the patient

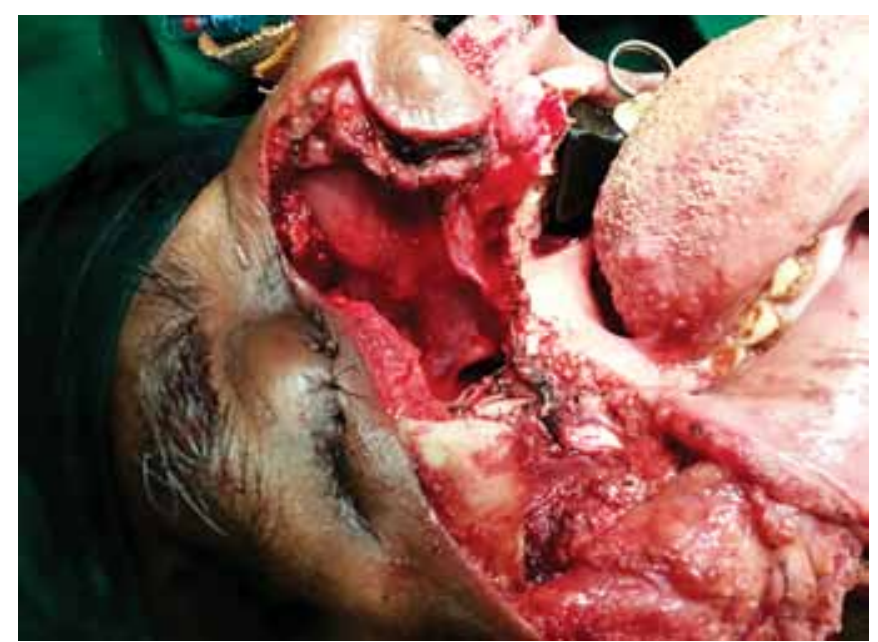

Fig. 2: Intraoperative photograph showing the defect after resection of the specimen

scanty cytoplasm which was periodic acid Schiff positive (Fig. 3). Later, immunohistochemistry for S-100 and CD 99 was done and found to be positive in this case.

Histopathology and the immunohistochemical studies confirmed the diagnosis of Ewing's Sarcoma.
After wound healing, an acrylic obturator was provided for the surgical defect.

Patient subsequently underwent postoperative radiotherapy and chemotherapy and is symptom free 15 months of follow-up period.

\section{DISCUSSION}

Ewing's sarcoma was first described by James Ewing in 1921. ${ }^{4}$ An extraskeletal form of this tumor was described by Angervall and Enzinger in $1975 .{ }^{5}$

The exact origin of the cells of Ewing's sarcoma has been a controversy. Recently, it has been found that Ewing's sarcoma is neuroectodermal in origin and is derived from cells of neural crest. ${ }^{1,4}$

There are two types of Ewing's sarcoma-osseous and extraosseous. Osseous type is more common and has predilection for long bones and pelvic girdle. The extraosseous type accounts for 4 to $7 \%$ of the tumors and affects the thigh, pelvis and prevertebral soft tissue. ${ }^{6}$

The precise diagnosis of these tumors is possible only after histological examination of the tumor tissue. The 


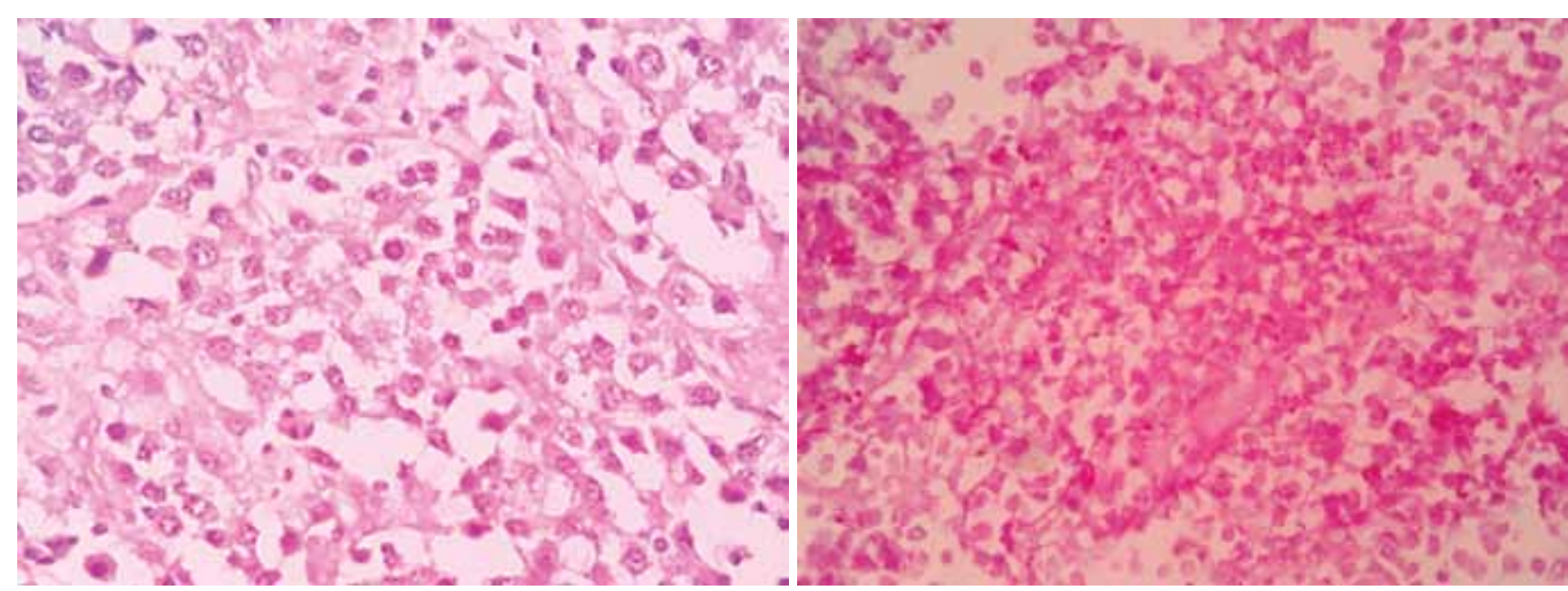

Fig. 3: Histopathology H\&E staining showing small uniform round cells vesicular nucleus with fine chromatin, with scanty cytoplasm which was periodic acid schiff positive as seen in Ewing's sarcoma which were CD99 positive in IHC

differential diagnosis includes lymphoma, rhabdomyosarcoma, neuroblastoma, reticulum cell sarcoma, small cell osteosarcoma and mesenchymal chondrosarcoma. ${ }^{4,6}$

Primitive neuroectodermal tumors (PNET) and Ewing's sarcoma form the two ends of the spectrum of neuroectodermal tumors, with Ewing's sarcoma representing the undifferentiated end whereas PNET forms the well differentiated end. ${ }^{2,7}$ It is difficult to differentiate between these two tumors because of the lack of specific immunohistochemical markers. ${ }^{6}$

The tumors in ESFT are treated on the basis of their clinical presentation (e.g. metastatic or localized). ${ }^{1}$

Surgery with wide margins followed by adjuvant radiotherapy and chemotherapy is the preferred treatment. ${ }^{6,4,8}$

In the present case, a complete surgical excision of the lesion was done with safe margins and patient underwent postoperative radiotherapy and chemotherapy in view of the locally advanced disease.

The prognosis appears to be dependent on the location of the primary tumor and the presence of distant metastasis. Survival rates in patients with Ewing's sarcoma of the head and neck region are significantly better than those patients with tumor in other locations. Less than $20 \%$ of the cases will have distant metastasis to lungs. Very rarely, it can metastasize to other bones and lymph nodes. ${ }^{6}$

\section{CONCLUSION}

Ewing's sarcoma is an aggressive malignancy which rarely affects the maxilla. However, treating clinician should have adequate knowledge of nonepithelial malignancies and should have adequate diagnostic work up, which includes immunohistochemistry. Initial treatment should be aggressive resection with wide margins. This gives good local control. It carries better prognosis than Ewing's sarcoma of the long bones as majority of these tumors in head and neck region do not metastasize early. In these cases, postoperative adjuvant treatment, regular follow-up and documentation is mandatory.

\section{REFERENCES}

1. Toretsky JA. Ewing sarcoma and primitive neuroectodermal tumours (last updated on: Jun 18, 2014). Available at: http:// www.emedicine.com/ped/topic2589.htm

2. Jayakumar S, Power D. Ewing's sarcoma/PNET: a histopathological review. Int J Orthoped Surg 2005;3(1):1-4.

3. Regezi JA, Sciuubba J. Oral pathology. Philadelphia: Saunders; 1993. Clinical-pathologic correlations; Ed3. p. 407-409.

4. Infante-Cossio P, Gutierrez-Perez JL, Garcia-Perla A, NoguerMediavilla M, Gavilan-Carrasco F. Primary Ewing's sarcoma of the maxilla and zygoma: report of a case. J Oral Maxillofac Surg 2005;63(10):1539-1542.

5. Cotterill SJ, Ahrens S, Paulussen M, Jurgens HF, Voute PA, Gadner H, Craft AW. Prognostic factors in Ewing's tumor of bone: analysis of 975 patients from the European intergroup cooperative Ewing's sarcoma study group. J Clin Oncol 2000; 18(17):3108-3114.

6. McMains KC, Gourin CG. Pathology-sarcomas of the head and neck (last updated on: Nov 1, 2013). Available at: http:// www.emedicine.com/ent/topic675.htm.

7. Votta TJ, Fantuzzo JJ, Boyd BC. Peripheral primitive neuroectodermal tumour associated with the anterior mandible: a case report and review of the literature. Oral Surg Oral Med Oral Pathol Oral Radiol 2005;100(5):592-597.

8. Postovsky S, Daitzchman M, Elhasid R, Arush MW. Ewing's sarcoma of the facial zygomatic area bones in a young child: a case presentation. Am J Otolaryngol 2000 May-Jun;21(3):213-215. 